\title{
Crystal Structure of $\mathrm{BaGe}\left[\mathrm{Ge}_{3} \mathrm{O}_{9}\right]$ and Its Relation to Benitoite
}

\author{
C. Robbins, A. Perloff, and S. Block \\ Institute for Materials Research, National Bureau of Standards, Washington, D.C. 20234
}

(May 23, 1966)

\begin{abstract}
$\mathrm{BaGe}\left[\mathrm{Ge}_{3} \mathrm{O}_{9}\right]$ is trigonal, space group P3, with lattice constants $a=11.61, c=4.74 \AA$, and $Z=3$.
The structure was established by three-dimensional Patterson and electron density syntheses. Three-dimensional least-squares refinement resulted in a final $\mathrm{R}$ value of 6.8 percent (observed data only).

The previously proposed structural relationship of this compound with benitoite, $\mathrm{BaTiSi}_{3} \mathrm{O}_{9}$, has been confirmed. The structure can be considered as composed of $\mathrm{Ge}_{3} \mathrm{O}_{9}$ rings, in which the $\mathrm{Ge}$ is tetrahedrally coordinated, linked through octahedrally coordinated $\mathrm{Ge}$ atoms to form a three-dimensional Ge-O network. All Ge polyhedra are linked by corner sharing. The Ba ions occupy positions in channels of the network.
\end{abstract}

Key Words: Barium tetragermanate, structure, benitoite, crystal, x-ray.

\section{Introduction}

The synthesis of three germanates of formula type $\mathrm{MeGe}_{4} \mathrm{O}_{9}(\mathrm{Me}=\mathrm{Sr}, \mathrm{Pb}, \mathrm{Ba})$ was reported by Robbins and Levin [1]. ${ }^{1}$ A comparison of indexed powder patterns suggested that the compounds were isostructural. This was later confirmed by Eulenberger, Wittman, and Nowotny [2]. In addition, they reported the synthesis of two forms of $\mathrm{CaGe}_{4} \mathrm{O}_{9}$ designated $\alpha$ and $\beta$, and found the $\alpha$ form was isostructural with $(\mathrm{Sr}, \mathrm{Pb}, \mathrm{Ba}) \mathrm{Ge}_{4} \mathrm{O}_{9}$.

Crystal chemical considerations (Robbins and Levin [1]) suggested a structural relationship with the mineral benitoite, $\mathrm{BaTiSi}_{3} \mathrm{O}_{9}$, whose structure was determined by Zachariasen [3]. Comparison of patterns of 1:4 germanates with indexed benitoite powder data supported this view. From these observations and a consideration of unit cell dimensions, a trial structure for the tetragermanates was obtained (Robbins and Levin [1]). To test the validity of the proposed model, the structure of $\mathrm{BaO} \cdot 4 \mathrm{GeO}_{2}$ was determined.

\section{Experimental Data}

Single crystals in the form of needles elongated along [001] were obtained by slowly cooling a melt of composition $\mathrm{BaGe}_{4} \mathrm{O}_{9}$ from slightly above the congruent melting point of $1392 \pm 5{ }^{\circ} \mathrm{C}$.

Unit cell dimensions, density measurements, and optical data for this compound were reported by

\footnotetext{
${ }^{1}$ Figures in brackets indicate the literature references at the end of this paper
}

Robbins and Levin [1]. Their data plus the space group information are:

$$
\begin{aligned}
a & =11.61 \AA \\
c & =4.74 \AA \\
S . G . & =\mathrm{P} 3 \\
Z & =3
\end{aligned}
$$$$
\begin{aligned}
\omega & =1.797 \pm 0.003 \\
\epsilon & =1.783 \pm 0.003 \\
\rho(\text { obs }) & =5.1 \mathrm{gcm}^{-3} \\
\rho(\text { calc }) & =5.12 \mathrm{gcm}^{-3}
\end{aligned}
$$

No systematic absences were observed. Precession films were consistent with space groups $\mathrm{P} 3 \mathrm{ml}$ or $\mathrm{P} \overline{3} \mathrm{ml}$. No satisfactory structure could be derived in these space groups. The possible space groups P3 or P $\overline{3}$ were then considered. The centrosymmetric choice could be eliminated on the basis of packing considerations because of the short $c$ dimension. The assumption that the space group is P3 was confirmed by the final structure.

Integrating Weissenberg films of levels $h k l$ with $l=0,1,2,3,4$ were taken with $\mathrm{Zr}$-filtered MoK $\alpha$ radiation $(\lambda=0.7107 \AA)$ using the multiple film technique. Intensities were measured with a densitometer comparator. Very weak reflections were estimated visually. The intensity data were obtained from a crystal approximately rectangular in cross section with dimensions of $0.043 \mathrm{~mm}, 0.066 \mathrm{~mm}$, and a length of $0.189 \mathrm{~mm}$. The linear absorption coefficient for molybdenum radiation is $222.41 \mathrm{~cm}^{-1}$.

Lorentz and polarization factor corrections were applied. Since the main objective of the study was the determination of positional parameters, no corrections for absorption errors were made. The latter would be expected to affect, primarily, the thermal parameters. The data consisted of 327 observed and 435 unobserved independent reflections. 
TABLE 1. Final atomic parameters ${ }^{\mathrm{a}}$

\begin{tabular}{l|r|r|r|r|r|r|r|r}
\hline \hline & $x$ & $\sigma(x)$ & $Y$ & $\sigma(Y)$ & \multicolumn{1}{c|}{$z$} & $\sigma(z)$ & \multicolumn{1}{c}{$\mathrm{B}$} & $\sigma(\mathrm{B})$ \\
\cline { 2 - 8 } & & & & & & & \\
$\mathrm{Ba}$ & 0.3341 & 0.0004 & 0.3334 & 0.0005 & \multicolumn{1}{c|}{0.0} & & 1.0265 & 0.0526 \\
$\mathrm{Ge}(1)$ & .0 & & .0 & & -.0928 & 0.0034 & 0.3509 & .2221 \\
$\mathrm{Ge}(2)$ & .6667 & & .3333 & & .0062 & .0038 & .5501 & .1798 \\
$\mathrm{Ge}(3)$ & .3333 & & .6667 & & .1024 & .0031 & .2158 & .1867 \\
$\mathrm{Ge}(4)$ & .8148 & .0007 & .8200 & .0006 & .4111 & .0025 & .3859 & .1570 \\
$\mathrm{Ge}(5)$ & .6674 & .0007 & .5131 & .0006 & .5051 & .0034 & .1402 & .0864 \\
$\mathrm{Ge}(6)$ & .5180 & .0006 & .6719 & .0007 & .5929 & .0025 & .3680 & .1275 \\
$\mathrm{O}(1)$ & .8459 & .0032 & .9286 & .0035 & .1193 & .0075 & -.4341 & .4651 \\
$\mathrm{O}(2)$ & .5843 & .0046 & .4074 & .0047 & .2158 & .0106 & 1.1598 & .7527 \\
$\mathrm{O}(3)$ & .7746 & .0033 & .6524 & .0033 & .3101 & .0078 & -0.1996 & .5040 \\
$\mathrm{O}(4)$ & .5505 & .0035 & .5561 & .0035 & .6965 & .0080 & .0655 & .5479 \\
$\mathrm{O}(5)$ & .4743 & .0056 & .7350 & .0058 & .8978 & .0127 & 2.2502 & .9361 \\
$\mathrm{O}(6)$ & .9264 & .0038 & .8428 & .0036 & .6430 & .0082 & 0.0008 & .5266 \\
$\mathrm{O}(7)$ & .6650 & .0069 & .8081 & .0068 & .4927 & .0175 & 3.0732 & 1.2087 \\
$\mathrm{O}(8)$ & .4059 & .0043 & .5880 & .0041 & .3274 & .0091 & 1.0008 & 0.6433 \\
$\mathrm{O}(9)$ & .7525 & .0048 & .4844 & .0046 & .7472 & .0103 & 1.1328 & .7087 \\
\hline
\end{tabular}

a The standard deviations are derived from the full-matrix least-squares refinement.

\section{Structure Determination}

Similarities in the x-ray powder patterns and unit cell dimensions of benitoite $\left(\mathrm{BaTiSi}_{3} \mathrm{O}_{9}\right)$ and $\mathrm{BaO} \cdot 4 \mathrm{GeO}_{2}$ led Robbins and Levin [1] to suggest that barium germanate probably exists as $\mathrm{BaGeGe}_{3} \mathrm{O}_{9}$ in a structural arrangement closely related to that found for benitoite by Zachariasen [3]. The large number of unobserved reflections obtained in the present study was consistent with the proposed relationship provided the $\mathrm{Ba}$ and $\mathrm{Ge}$ atoms had $x$ and $y$ parameters near $1 / 3$ and $2 / 3$ and $z$ parameters near 0 or $1 / 2$ in the germanate cei. In benitoite $\mathrm{Ba}, \mathrm{Ti}$, and the $\mathrm{Si}_{3} \mathrm{O}_{9}$ rings all are on, or around threefold axes. The lower symmetry of the germanate permits, at most, only one of the corresponding atoms or groups to be on a threefold axis.

A three-dimensional Patterson function was computed and peaks corresponding to $\mathrm{Ba}-\mathrm{Ba}$ and $\mathrm{Ge}-\mathrm{Ge}$ vectors were identified and related to the proposed model. On the basis of the trial structure and space group P3, three choices of origin were possible i.e., at the center of a $\mathrm{Ge}_{3} \mathrm{O}_{9}$ group, at a $\mathrm{Ba}$ atom or at an octahedrally coordinated Ge atom. Using coordinates of $\mathrm{Ba}$ and $\mathrm{Ge}$ from the model, three cycles of Fourier refinement were calculated for each choice of origin. Only the model with a $\mathrm{Ge}$ atom at the origin on a threefold axis refined satisfactorily. Using phases based on $\mathrm{Ba}$ and $\mathrm{Ge}$ positions from the Fourier refinement, a 3-dimensional electron density map was calculated which yielded the oxygen coordinates.

The structure was refined by a full-matrix leastsquares analysis of the 327 observed and 435 unobserved reflections. The final conventional $R$ value based on the 327 observed independent reflections was 0.068 . The total number of parameters varied was 62 which included $x, y$, and $z$, an isotropic temperature factor for each atom and a scale factor for each level. The final parameters from this refinement are listed in table 1 . The temperature factors are not considered meaningful, primarily because of absorption. The correlation matrix from this refinement indicated that many of the variables were correlated to a moderate degree (correlation coefficients on the order of 0.5 to 0.6). The possibility that this was the source of the unrealistic temperature factors was considered. A second least-squares refinement was made in which, alternately, scale and position parameters were varied for 2 cycles with fixed temperature factors and then temperature factors were varied for 2 cycles with fixed scale and position parameters. This was repeated for a total of eight cycles. The result was identical with the first refinement to within one standard deviation. Therefore, only the parameters from the first refinement are given in this paper. Observed and calculated structure factors are reported in table 2 .

Atomic scattering factors for neutral $\mathrm{Ge}$ and $0^{-1}$ were taken from International Tables for X-ray Crystallography (1962). Values for $\mathrm{Ba}^{+2}$ were taken from Thomas and Umeda [4]. The barium and germanium form factors were corrected for dispersion (International Tables for X-ray Crystallography, 1962).

\section{Structure Description}

A projection of the structure of $\mathrm{BaO} \cdot 4 \mathrm{GeO}_{2}$ along [001] is shown in figure 1. Numbering of the atoms of the asymmetric unit is consistent with table 1 . The structure is made up of rings of three $\mathrm{GeO}_{4}$ tetrahedra linked together by $\mathrm{GeO}_{6}$ octahedra. Barium atoms are located in channels of the network. Germanium atoms 1,2, and 3 are octahedrally coordinated and lie on threefold axes. Germanium atoms 4,5 , and 6 , in tetrahedral coordination, make up the $\mathrm{Ge}_{3} \mathrm{O}_{9}$ ring. Six rings are linked through a germanium octahedron to form the germanium-oxygen network. The two nonring oxygens of each of three equivalent $\mathrm{GeO}_{4}$ tetrahedra are bonded to germanium atoms above and below the ring to form $\mathrm{GeO}_{6}$ groups. Every germanium polyhedron shares all of its corners: $\mathrm{GeO}_{4}$ tetrahedra share only corners with other polyhedra with the exception of $\mathrm{Ge}(6)$, which shares one edge with a barium polyhedron; each germanium octahedron shares three edges with three different barium polyhedra.

The $\mathrm{Ge}_{3} \mathrm{O}_{9}$ ring, projected along [001], is shown in figure 2. Interatomic distances, angles and standard deviations for the three germanium atoms in tetra- 


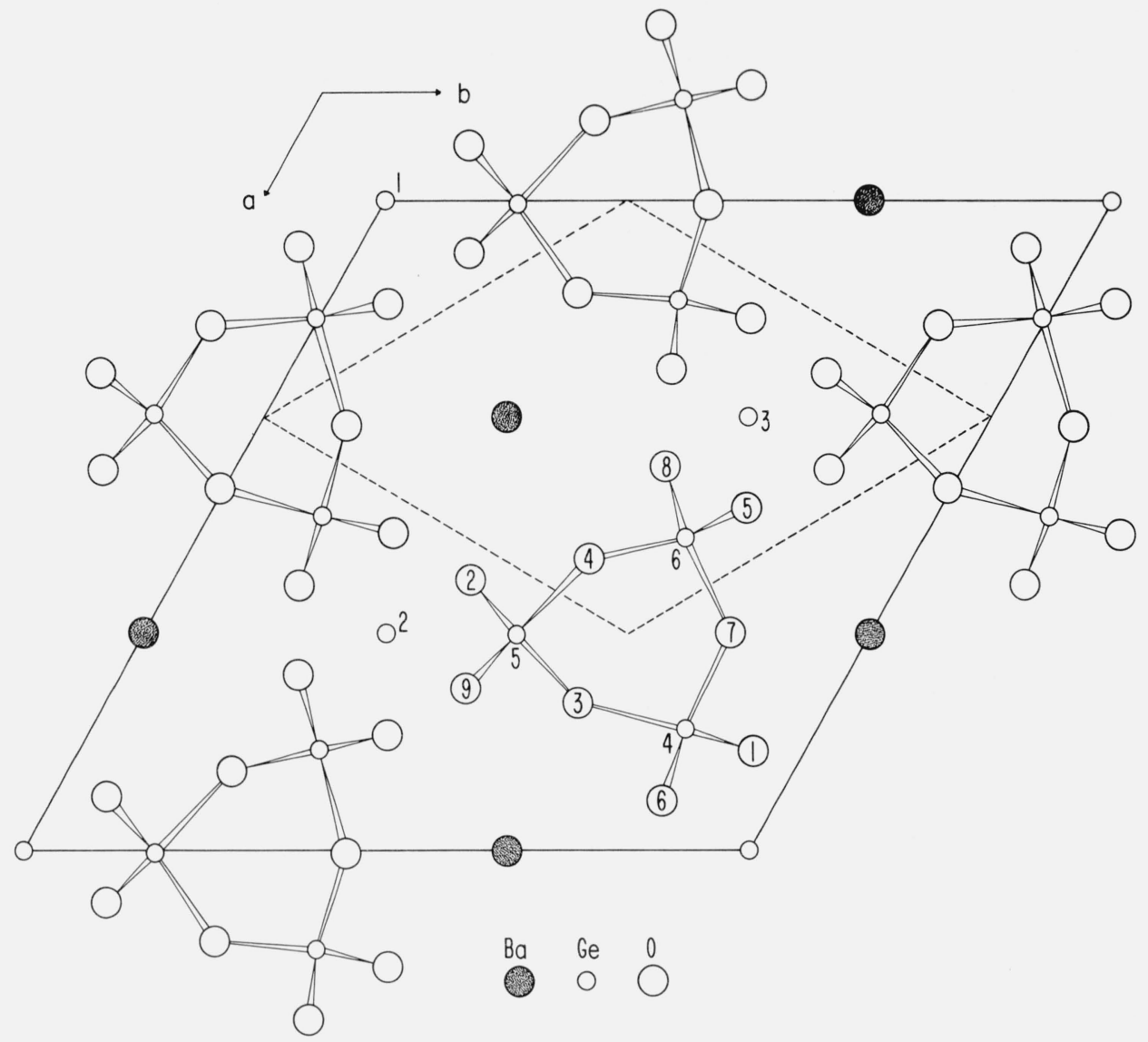

Figure 1. [001] Projection of the $\mathrm{BaGe}\left[\mathrm{Ge}_{3} \mathrm{O}_{9}\right]$ structure. The numbering is that of table 1 .

Figure 2. [001] Projection of the $\mathrm{Ge}_{3} \mathrm{O}_{9}$ ring. The numbers identify atoms in table 1 . Bond distances are in $\AA$.

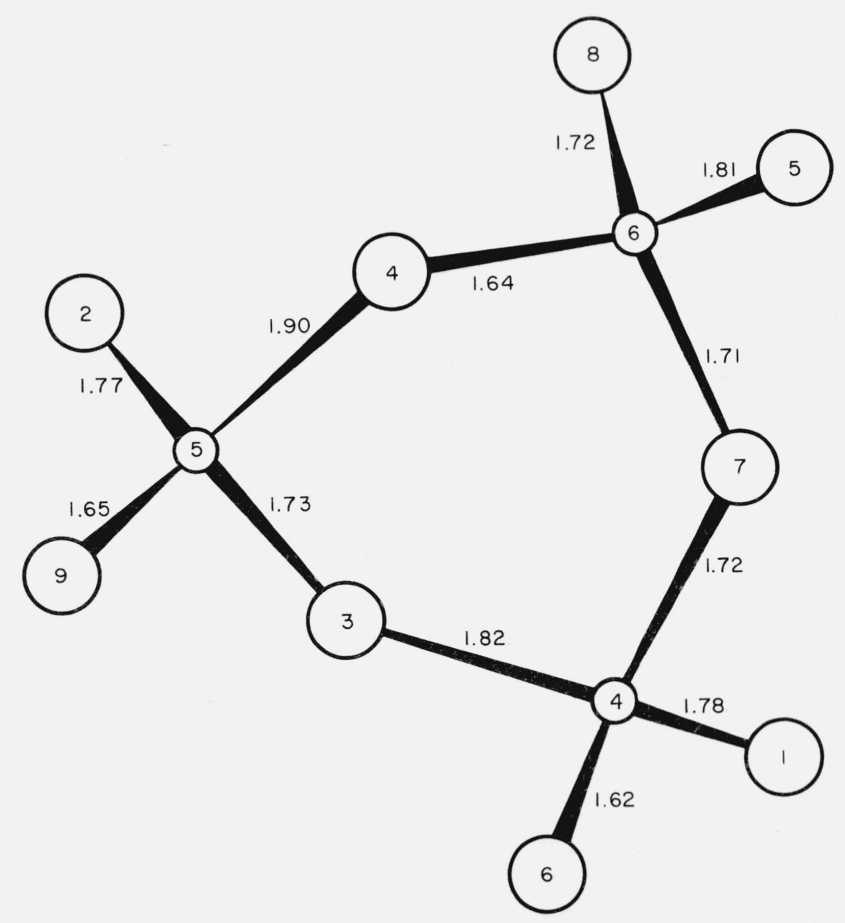


TABLE 2. Comparison of observed and calculated structure factors $\mathrm{S}(\mathrm{hk} 0)=1.97, \mathrm{~S}(\mathrm{hk} 1)=2.68$, $\mathrm{S}(\mathrm{hk} 2)=3.09, \mathrm{~S}(\mathrm{hk} 3)=3.25, \mathrm{~S}(\mathrm{hk} 4)=4.44$

\begin{tabular}{|c|c|c|c|c|c|c|c|c|c|c|c|c|c|c|}
\hline$h$ & $k$ & $l$ & Fo & $\mathrm{sFc}$ & $h$ & $k$ & $l$ & Fo & $\mathrm{sFc}$ & $h$ & $k$ & $l$ & $F_{0}$ & $\mathrm{sFc}$ \\
\hline $\begin{array}{l}0 \\
1 \\
1 \\
1 \\
1\end{array}$ & $\begin{array}{r}15 \\
4 \\
-5 \\
7 \\
-8\end{array}$ & $\begin{array}{l}0 \\
0 \\
0 \\
0 \\
0\end{array}$ & $\begin{array}{r}97 \\
104 \\
111 \\
55 \\
53\end{array}$ & $\begin{array}{r}88 \\
107 \\
108 \\
58 \\
50\end{array}$ & $\begin{array}{r}3 \\
3 \\
-3 \\
3 \\
-3\end{array}$ & $\begin{array}{r}5 \\
6 \\
-6 \\
-7 \\
7\end{array}$ & $\begin{array}{l}1 \\
1 \\
1 \\
1 \\
1\end{array}$ & $\begin{array}{r}55 \\
178 \\
190 \\
51 \\
43\end{array}$ & $\begin{array}{r}68 \\
170 \\
184 \\
73 \\
46\end{array}$ & $\begin{array}{r}2 \\
-2 \\
-2 \\
3 \\
3\end{array}$ & $\begin{array}{r}8 \\
0 \\
10 \\
0 \\
-1\end{array}$ & $\begin{array}{l}2 \\
2 \\
2 \\
2 \\
2\end{array}$ & $\begin{array}{r}124 \\
74 \\
124 \\
136 \\
103\end{array}$ & $\begin{array}{r}121 \\
81 \\
120 \\
133 \\
95\end{array}$ \\
\hline $\begin{array}{l}1 \\
1 \\
2 \\
2 \\
2\end{array}$ & $\begin{array}{r}10 \\
-11 \\
5 \\
-7 \\
8\end{array}$ & $\begin{array}{l}0 \\
0 \\
0 \\
0 \\
0\end{array}$ & $\begin{array}{r}94 \\
92 \\
60 \\
60 \\
169\end{array}$ & $\begin{array}{r}94 \\
95 \\
49 \\
57 \\
171\end{array}$ & $\begin{array}{r}-3 \\
3 \\
-3 \\
-3 \\
4\end{array}$ & $\begin{array}{r}-9 \\
9 \\
9 \\
12 \\
0\end{array}$ & $\begin{array}{l}1 \\
1 \\
1 \\
1 \\
1\end{array}$ & $\begin{array}{r}156 \\
165 \\
176 \\
168 \\
34\end{array}$ & $\begin{array}{r}153 \\
155 \\
173 \\
160 \\
36\end{array}$ & $\begin{array}{l}3 \\
3 \\
3 \\
3 \\
3\end{array}$ & $\begin{array}{r}-2 \\
2 \\
4 \\
5 \\
-6\end{array}$ & $\begin{array}{l}2 \\
2 \\
2 \\
2 \\
2\end{array}$ & $\begin{array}{r}62 \\
72 \\
55 \\
53 \\
107\end{array}$ & $\begin{array}{r}60 \\
54 \\
39 \\
53 \\
109\end{array}$ \\
\hline $\begin{array}{r}-2 \\
2 \\
-2 \\
2 \\
-2\end{array}$ & $\begin{array}{l}10 \\
11 \\
13 \\
14 \\
16\end{array}$ & $\begin{array}{l}0 \\
0 \\
0 \\
0 \\
0\end{array}$ & $\begin{array}{r}169 \\
46 \\
42 \\
53 \\
54\end{array}$ & $\begin{array}{r}176 \\
49 \\
50 \\
56 \\
56\end{array}$ & $\begin{array}{r}4 \\
4 \\
4 \\
-4 \\
4\end{array}$ & $\begin{array}{r}-1 \\
1 \\
-2 \\
-4 \\
4\end{array}$ & $\begin{array}{l}1 \\
1 \\
1 \\
1 \\
1\end{array}$ & $\begin{array}{r}33 \\
147 \\
82 \\
75 \\
72\end{array}$ & $\begin{array}{r}38 \\
146 \\
94 \\
80 \\
81\end{array}$ & $\begin{array}{r}3 \\
3 \\
-3 \\
4 \\
4\end{array}$ & $\begin{array}{r}6 \\
-7 \\
9 \\
0 \\
1\end{array}$ & $\begin{array}{l}2 \\
2 \\
2 \\
2 \\
2\end{array}$ & $\begin{array}{r}84 \\
99 \\
90 \\
115 \\
73\end{array}$ & $\begin{array}{r}88 \\
98 \\
95 \\
105 \\
68\end{array}$ \\
\hline $\begin{array}{l}3 \\
3 \\
3 \\
3 \\
3\end{array}$ & $\begin{array}{r}0 \\
3 \\
6 \\
-9 \\
9\end{array}$ & $\begin{array}{l}0 \\
0 \\
0 \\
0 \\
0\end{array}$ & $\begin{array}{r}123 \\
116 \\
132 \\
134 \\
80\end{array}$ & $\begin{array}{r}113 \\
111 \\
138 \\
134 \\
85\end{array}$ & $\begin{array}{r}-4 \\
4 \\
-4 \\
4 \\
5\end{array}$ & $\begin{array}{l}5 \\
7 \\
7 \\
9 \\
0\end{array}$ & $\begin{array}{l}1 \\
1 \\
1 \\
1 \\
1\end{array}$ & $\begin{array}{r}145 \\
92 \\
82 \\
50 \\
92\end{array}$ & $\begin{array}{r}150 \\
80 \\
83 \\
58 \\
83\end{array}$ & $\begin{array}{r}4 \\
4 \\
4 \\
-4 \\
5\end{array}$ & $\begin{array}{r}-2 \\
4 \\
6 \\
-7 \\
0\end{array}$ & $\begin{array}{l}2 \\
2 \\
2 \\
2 \\
2\end{array}$ & $\begin{array}{r}223 \\
164 \\
60 \\
72 \\
62\end{array}$ & $\begin{array}{r}224 \\
148 \\
56 \\
74 \\
66\end{array}$ \\
\hline $\begin{array}{l}3 \\
4 \\
4 \\
4 \\
4\end{array}$ & $\begin{array}{r}12 \\
4 \\
7 \\
10 \\
13\end{array}$ & $\begin{array}{l}0 \\
0 \\
0 \\
0 \\
0\end{array}$ & $\begin{array}{r}82 \\
208 \\
85 \\
120 \\
43\end{array}$ & $\begin{array}{r}77 \\
230 \\
75 \\
115 \\
50\end{array}$ & $\begin{array}{r}5 \\
5 \\
5 \\
5 \\
-5\end{array}$ & $\begin{array}{r}2 \\
-3 \\
3 \\
5 \\
-5\end{array}$ & $\begin{array}{l}1 \\
1 \\
1 \\
1 \\
1\end{array}$ & $\begin{array}{r}139 \\
56 \\
33 \\
108 \\
114\end{array}$ & $\begin{array}{r}130 \\
59 \\
37 \\
106 \\
104\end{array}$ & $\begin{array}{l}5 \\
5 \\
5 \\
5 \\
5\end{array}$ & $\begin{array}{r}-1 \\
1 \\
2 \\
-2 \\
5\end{array}$ & $\begin{array}{l}2 \\
2 \\
2 \\
2 \\
2\end{array}$ & $\begin{array}{r}61 \\
156 \\
73 \\
60 \\
72\end{array}$ & $\begin{array}{r}66 \\
146 \\
72 \\
57 \\
76\end{array}$ \\
\hline $\begin{array}{l}5 \\
5 \\
5 \\
6 \\
6\end{array}$ & $\begin{array}{r}5 \\
11 \\
-16 \\
6 \\
9\end{array}$ & $\begin{array}{l}0 \\
0 \\
0 \\
0 \\
0\end{array}$ & $\begin{array}{r}155 \\
100 \\
87 \\
189 \\
90\end{array}$ & $\begin{array}{r}156 \\
97 \\
90 \\
194 \\
84\end{array}$ & $\begin{array}{r}5 \\
-5 \\
-5 \\
6 \\
7\end{array}$ & $\begin{array}{r}8 \\
-8 \\
13 \\
3 \\
1\end{array}$ & $\begin{array}{l}1 \\
1 \\
1 \\
1 \\
1\end{array}$ & $\begin{array}{r}103 \\
86 \\
111 \\
193 \\
175\end{array}$ & $\begin{array}{r}94 \\
87 \\
95 \\
188 \\
171\end{array}$ & $\begin{array}{l}5 \\
6 \\
6 \\
6 \\
6\end{array}$ & $\begin{array}{r}7 \\
-1 \\
1 \\
-3 \\
3\end{array}$ & $\begin{array}{l}2 \\
2 \\
2 \\
2 \\
2\end{array}$ & $\begin{array}{r}76 \\
150 \\
37 \\
104 \\
66\end{array}$ & $\begin{array}{r}79 \\
150 \\
47 \\
106 \\
67\end{array}$ \\
\hline $\begin{array}{l}7 \\
7 \\
8 \\
9 \\
9\end{array}$ & $\begin{array}{r}4 \\
10 \\
8 \\
0 \\
3\end{array}$ & $\begin{array}{l}0 \\
0 \\
0 \\
0 \\
0\end{array}$ & $\begin{array}{r}81 \\
64 \\
79 \\
108 \\
79\end{array}$ & $\begin{array}{r}87 \\
65 \\
68 \\
107 \\
85\end{array}$ & $\begin{array}{r}-7 \\
7 \\
-7 \\
7 \\
8\end{array}$ & $\begin{array}{r}-4 \\
7 \\
-7 \\
-9 \\
1\end{array}$ & $\begin{array}{l}1 \\
1 \\
1 \\
1 \\
1\end{array}$ & $\begin{array}{r}77 \\
134 \\
134 \\
69 \\
56\end{array}$ & $\begin{array}{r}76 \\
125 \\
125 \\
83 \\
55\end{array}$ & $\begin{array}{r}6 \\
6 \\
6 \\
-6 \\
7\end{array}$ & $\begin{array}{r}-5 \\
5 \\
6 \\
6 \\
0\end{array}$ & $\begin{array}{l}2 \\
2 \\
2 \\
2 \\
2\end{array}$ & $\begin{array}{r}96 \\
109 \\
157 \\
215 \\
74\end{array}$ & $\begin{array}{r}83 \\
110 \\
150 \\
234 \\
77\end{array}$ \\
\hline $\begin{array}{r}9 \\
9 \\
10 \\
10 \\
12\end{array}$ & $\begin{array}{r}6 \\
9 \\
7 \\
10 \\
0\end{array}$ & $\begin{array}{l}0 \\
0 \\
0 \\
0 \\
0\end{array}$ & $\begin{array}{r}93 \\
93 \\
54 \\
50 \\
185\end{array}$ & $\begin{array}{r}90 \\
80 \\
60 \\
52 \\
179\end{array}$ & $\begin{array}{r}8 \\
8 \\
-8 \\
9 \\
9\end{array}$ & $\begin{array}{r}-5 \\
5 \\
8 \\
0 \\
-1\end{array}$ & $\begin{array}{l}1 \\
1 \\
1 \\
1 \\
1\end{array}$ & $\begin{array}{r}59 \\
103 \\
72 \\
140 \\
41\end{array}$ & $\begin{array}{r}66 \\
91 \\
66 \\
128 \\
60\end{array}$ & $\begin{array}{l}7 \\
7 \\
7 \\
7 \\
8\end{array}$ & $\begin{array}{r}-2 \\
-3 \\
3 \\
4 \\
1\end{array}$ & $\begin{array}{l}2 \\
2 \\
2 \\
2 \\
2\end{array}$ & $\begin{array}{r}63 \\
107 \\
73 \\
69 \\
51\end{array}$ & $\begin{array}{r}64 \\
101 \\
67 \\
70 \\
59\end{array}$ \\
\hline $\begin{array}{l}12 \\
12 \\
12 \\
12 \\
13\end{array}$ & $\begin{array}{r}3 \\
6 \\
9 \\
-18 \\
4\end{array}$ & $\begin{array}{l}0 \\
0 \\
0 \\
0 \\
0\end{array}$ & $\begin{array}{r}79 \\
113 \\
51 \\
86 \\
49\end{array}$ & $\begin{array}{l}75 \\
93 \\
50 \\
82 \\
54\end{array}$ & $\begin{array}{r}9 \\
-9 \\
9 \\
9 \\
10\end{array}$ & $\begin{array}{r}-2 \\
-6 \\
6 \\
-7 \\
1\end{array}$ & $\begin{array}{l}1 \\
1 \\
1 \\
1 \\
1\end{array}$ & $\begin{array}{l}56 \\
84 \\
89 \\
62 \\
81\end{array}$ & $\begin{array}{l}60 \\
89 \\
74 \\
78 \\
81\end{array}$ & $\begin{array}{l}8 \\
8 \\
8 \\
9 \\
9\end{array}$ & $\begin{array}{r}2 \\
-4 \\
-5 \\
0 \\
-1\end{array}$ & $\begin{array}{l}2 \\
2 \\
2 \\
2 \\
2\end{array}$ & $\begin{array}{r}118 \\
167 \\
67 \\
108 \\
51\end{array}$ & $\begin{array}{r}121 \\
170 \\
61 \\
93 \\
53\end{array}$ \\
\hline $\begin{array}{r}14 \\
14 \\
15 \\
0 \\
0\end{array}$ & $\begin{array}{r}-7 \\
-10 \\
3 \\
3 \\
4\end{array}$ & $\begin{array}{l}0 \\
0 \\
0 \\
1 \\
1\end{array}$ & $\begin{array}{r}36 \\
116 \\
52 \\
274 \\
92\end{array}$ & $\begin{array}{r}45 \\
105 \\
65 \\
286 \\
89\end{array}$ & $\begin{array}{r}10 \\
11 \\
-12 \\
-12 \\
13\end{array}$ & $\begin{array}{r}3 \\
-1 \\
-3 \\
9 \\
-3\end{array}$ & $\begin{array}{l}1 \\
1 \\
1 \\
1 \\
1\end{array}$ & $\begin{array}{r}42 \\
90 \\
103 \\
170 \\
51\end{array}$ & $\begin{array}{r}48 \\
87 \\
98 \\
157 \\
52\end{array}$ & $\begin{array}{r}9 \\
9 \\
9 \\
10 \\
-10\end{array}$ & $\begin{array}{r}3 \\
-3 \\
-10 \\
1 \\
-1\end{array}$ & $\begin{array}{l}2 \\
2 \\
2 \\
2 \\
2\end{array}$ & $\begin{array}{l}66 \\
68 \\
72 \\
50 \\
92\end{array}$ & $\begin{array}{l}67 \\
65 \\
65 \\
50 \\
82\end{array}$ \\
\hline $\begin{array}{l}0 \\
0 \\
1 \\
1 \\
1\end{array}$ & $\begin{array}{l}5 \\
9 \\
2 \\
3 \\
4\end{array}$ & $\begin{array}{l}1 \\
1 \\
1 \\
1 \\
1\end{array}$ & $\begin{array}{r}35 \\
109 \\
85 \\
29 \\
127\end{array}$ & $\begin{array}{r}42 \\
130 \\
80 \\
31 \\
128\end{array}$ & 0 & $\begin{array}{l}2 \\
3 \\
4 \\
5 \\
6\end{array}$ & $\begin{array}{l}2 \\
2 \\
2 \\
2 \\
2\end{array}$ & $\begin{array}{r}36 \\
114 \\
37 \\
188 \\
229\end{array}$ & $\begin{array}{r}33 \\
109 \\
40 \\
190 \\
233\end{array}$ & $\begin{array}{l}10 \\
10 \\
10 \\
10 \\
10\end{array}$ & $\begin{array}{l}-2 \\
-3 \\
-5 \\
-6 \\
-9\end{array}$ & $\begin{array}{l}2 \\
2 \\
2 \\
2 \\
2\end{array}$ & $\begin{array}{r}135 \\
62 \\
51 \\
51 \\
54\end{array}$ & $\begin{array}{r}125 \\
64 \\
60 \\
56 \\
63\end{array}$ \\
\hline $\begin{array}{r}-1 \\
1 \\
-1 \\
-1 \\
1\end{array}$ & $\begin{array}{r}5 \\
7 \\
-7 \\
8 \\
13\end{array}$ & $\begin{array}{l}1 \\
1 \\
1 \\
1 \\
1\end{array}$ & $\begin{array}{r}129 \\
164 \\
171 \\
171 \\
79\end{array}$ & $\begin{array}{r}125 \\
160 \\
166 \\
169 \\
94\end{array}$ & $\begin{array}{l}1 \\
1 \\
1\end{array}$ & $\begin{array}{r}9 \\
11 \\
1 \\
3 \\
4\end{array}$ & $\begin{array}{l}2 \\
2 \\
2 \\
2 \\
2\end{array}$ & $\begin{array}{l}76 \\
94 \\
62 \\
99 \\
83\end{array}$ & $\begin{array}{r}71 \\
103 \\
63 \\
97 \\
75\end{array}$ & $\begin{array}{r}-10 \\
-11 \\
11 \\
11 \\
11\end{array}$ & $\begin{array}{r}10 \\
1 \\
1 \\
-1 \\
2\end{array}$ & $\begin{array}{l}2 \\
2 \\
2 \\
2 \\
2\end{array}$ & $\begin{array}{l}93 \\
82 \\
69 \\
77 \\
64\end{array}$ & $\begin{array}{l}89 \\
77 \\
74 \\
66 \\
56\end{array}$ \\
\hline $\begin{array}{r}2 \\
2 \\
2 \\
-2 \\
-2\end{array}$ & $\begin{array}{r}1 \\
-1 \\
2 \\
3 \\
-3\end{array}$ & $\begin{array}{l}1 \\
1 \\
1 \\
1 \\
1\end{array}$ & $\begin{array}{r}105 \\
206 \\
97 \\
107 \\
72\end{array}$ & $\begin{array}{r}100 \\
216 \\
94 \\
94 \\
66\end{array}$ & $\begin{array}{r}-1 \\
1 \\
-1 \\
1 \\
-1\end{array}$ & $\begin{array}{l}4 \\
5 \\
5 \\
6 \\
7\end{array}$ & $\begin{array}{l}2 \\
2 \\
2 \\
2 \\
2\end{array}$ & $\begin{array}{r}107 \\
86 \\
79 \\
104 \\
106\end{array}$ & $\begin{array}{l}99 \\
85 \\
83 \\
98 \\
96\end{array}$ & $\begin{array}{r}11 \\
12 \\
12 \\
-12 \\
12\end{array}$ & $\begin{array}{r}-5 \\
0 \\
-1 \\
3 \\
-3\end{array}$ & $\begin{array}{l}2 \\
2 \\
2 \\
2 \\
2\end{array}$ & $\begin{array}{r}105 \\
119 \\
87 \\
89 \\
78\end{array}$ & $\begin{array}{r}107 \\
116 \\
73 \\
79 \\
65\end{array}$ \\
\hline $\begin{array}{r}2 \\
-2 \\
2 \\
2 \\
-2\end{array}$ & $\begin{array}{r}3 \\
-5 \\
5 \\
6 \\
7\end{array}$ & $\begin{array}{l}1 \\
1 \\
1 \\
1 \\
1\end{array}$ & $\begin{array}{r}70 \\
130 \\
146 \\
37 \\
141\end{array}$ & $\begin{array}{r}64 \\
127 \\
143 \\
44 \\
136\end{array}$ & $\begin{array}{l}1 \\
1 \\
2 \\
2 \\
2\end{array}$ & $\begin{array}{r}9 \\
10 \\
0 \\
-1 \\
2\end{array}$ & $\begin{array}{l}2 \\
2 \\
2 \\
2 \\
2\end{array}$ & $\begin{array}{r}54 \\
65 \\
40 \\
79 \\
198\end{array}$ & $\begin{array}{r}65 \\
77 \\
34 \\
78 \\
214\end{array}$ & $\begin{array}{r}12 \\
12 \\
12 \\
0\end{array}$ & $\begin{array}{r}-6 \\
-7 \\
-9 \\
2 \\
3\end{array}$ & $\begin{array}{l}2 \\
2 \\
2 \\
2 \\
3\end{array}$ & $\begin{array}{r}149 \\
71 \\
89 \\
38 \\
145\end{array}$ & $\begin{array}{r}143 \\
80 \\
77 \\
42 \\
147\end{array}$ \\
\hline $\begin{array}{r}-2 \\
2 \\
3 \\
3 \\
3\end{array}$ & $\begin{array}{r}8 \\
11 \\
0 \\
2 \\
4\end{array}$ & $\begin{array}{l}1 \\
1 \\
1 \\
1 \\
1\end{array}$ & $\begin{array}{r}40 \\
47 \\
239 \\
68 \\
39\end{array}$ & $\begin{array}{r}43 \\
55 \\
253 \\
66 \\
57\end{array}$ & $\begin{array}{r}2 \\
2 \\
-2 \\
2 \\
-2\end{array}$ & $\begin{array}{l}4 \\
5 \\
6 \\
7 \\
7\end{array}$ & $\begin{array}{l}2 \\
2 \\
2 \\
2 \\
2\end{array}$ & $\begin{array}{l}66 \\
47 \\
54 \\
78 \\
45\end{array}$ & $\begin{array}{l}61 \\
57 \\
58 \\
80 \\
39\end{array}$ & $\begin{array}{l}1 \\
1\end{array}$ & $\begin{array}{l}5 \\
6 \\
8 \\
1 \\
3\end{array}$ & $\begin{array}{l}3 \\
3 \\
3 \\
3 \\
3\end{array}$ & $\begin{array}{r}89 \\
63 \\
88 \\
97 \\
148\end{array}$ & $\begin{array}{r}95 \\
60 \\
90 \\
108 \\
145\end{array}$ \\
\hline
\end{tabular}


TABLE 2. Comparison of observed and calculated structure factors $\mathrm{S}(\mathrm{hk} 0=1.97, \mathrm{~S}(\mathrm{hk} \mathrm{l})=2.68, \mathrm{~S}(\mathrm{hk} 2)$ $=3.09, \mathrm{~S}(\mathrm{hk} 3)=3.25, \mathrm{~S}(\mathrm{hk} 4)=4.44-$ Continued

\begin{tabular}{|c|c|c|c|c|c|c|c|c|c|c|c|c|c|c|}
\hline$h$ & $k$ & $l$ & Fo & $\mathrm{sFc}$ & $h$ & $k$ & $l$ & Fo & $\mathrm{sFc}$ & $h$ & $k$ & $l$ & Fo & $\mathrm{sFc}$ \\
\hline $\begin{array}{r}1 \\
1 \\
-1 \\
2 \\
2\end{array}$ & $\begin{array}{r}4 \\
7 \\
8 \\
0 \\
-1\end{array}$ & $\begin{array}{l}3 \\
3 \\
3 \\
3 \\
3\end{array}$ & $\begin{array}{r}93 \\
82 \\
82 \\
17 \\
112\end{array}$ & $\begin{array}{r}87 \\
76 \\
82 \\
26 \\
107\end{array}$ & $\begin{array}{l}8 \\
8 \\
8 \\
9 \\
9\end{array}$ & $\begin{array}{r}-1 \\
-2 \\
-4 \\
0 \\
-1\end{array}$ & $\begin{array}{l}3 \\
3 \\
3 \\
3 \\
3\end{array}$ & $\begin{array}{r}82 \\
79 \\
41 \\
93 \\
122\end{array}$ & $\begin{array}{r}80 \\
82 \\
47 \\
87 \\
121\end{array}$ & $\begin{array}{l}4 \\
4 \\
5 \\
5 \\
5\end{array}$ & $\begin{array}{r}-3 \\
4 \\
1 \\
-2 \\
4\end{array}$ & $\begin{array}{l}4 \\
4 \\
4 \\
4 \\
4\end{array}$ & $\begin{array}{r}90 \\
64 \\
131 \\
35 \\
74\end{array}$ & $\begin{array}{r}80 \\
68 \\
128 \\
35 \\
74\end{array}$ \\
\hline $\begin{array}{l}2 \\
2 \\
2 \\
2 \\
2\end{array}$ & $\begin{array}{r}1 \\
2 \\
5 \\
7 \\
-7\end{array}$ & $\begin{array}{l}3 \\
3 \\
3 \\
3 \\
3\end{array}$ & $\begin{array}{r}186 \\
53 \\
93 \\
157 \\
79\end{array}$ & $\begin{array}{r}172 \\
51 \\
90 \\
144 \\
79\end{array}$ & $\begin{array}{r}9 \\
9 \\
9 \\
10 \\
10\end{array}$ & $\begin{array}{r}3 \\
-3 \\
-9 \\
1 \\
3\end{array}$ & $\begin{array}{l}3 \\
3 \\
3 \\
3 \\
3\end{array}$ & $\begin{array}{r}49 \\
100 \\
78 \\
77 \\
98\end{array}$ & $\begin{array}{r}61 \\
105 \\
82 \\
67 \\
98\end{array}$ & $\begin{array}{l}5 \\
5 \\
7 \\
7 \\
7\end{array}$ & $\begin{array}{r}-4 \\
5 \\
1 \\
3 \\
4\end{array}$ & $\begin{array}{l}4 \\
4 \\
4 \\
4 \\
4\end{array}$ & $\begin{array}{l}90 \\
73 \\
67 \\
68 \\
45\end{array}$ & $\begin{array}{l}80 \\
69 \\
55 \\
64 \\
58\end{array}$ \\
\hline $\begin{array}{r}-2 \\
-2 \\
3 \\
3 \\
3\end{array}$ & $\begin{array}{r}7 \\
9 \\
0 \\
-1 \\
2\end{array}$ & $\begin{array}{l}3 \\
3 \\
3 \\
3 \\
3\end{array}$ & $\begin{array}{r}96 \\
141 \\
99 \\
170 \\
127\end{array}$ & $\begin{array}{r}89 \\
143 \\
93 \\
175 \\
115\end{array}$ & $\begin{array}{l}10 \\
10 \\
11 \\
11 \\
11\end{array}$ & $\begin{array}{l}-3 \\
-5 \\
-1 \\
-4 \\
-5\end{array}$ & $\begin{array}{l}3 \\
3 \\
3 \\
3 \\
3\end{array}$ & $\begin{array}{r}109 \\
85 \\
80 \\
41 \\
75\end{array}$ & $\begin{array}{r}105 \\
77 \\
63 \\
48 \\
67\end{array}$ & $\begin{array}{l}7 \\
7 \\
8 \\
8 \\
8\end{array}$ & $\begin{array}{r}-5 \\
-8 \\
1 \\
-1 \\
-4\end{array}$ & $\begin{array}{l}4 \\
4 \\
4 \\
4 \\
4\end{array}$ & $\begin{array}{l}65 \\
67 \\
93 \\
67 \\
52\end{array}$ & $\begin{array}{l}61 \\
73 \\
76 \\
58 \\
65\end{array}$ \\
\hline $\begin{array}{r}3 \\
3 \\
3 \\
-3 \\
-3\end{array}$ & $\begin{array}{r}3 \\
5 \\
-7 \\
8 \\
9\end{array}$ & $\begin{array}{l}3 \\
3 \\
3 \\
3 \\
3\end{array}$ & $\begin{array}{r}108 \\
103 \\
167 \\
105 \\
80\end{array}$ & $\begin{array}{r}106 \\
104 \\
159 \\
105 \\
74\end{array}$ & $\begin{array}{r}11 \\
12 \\
12 \\
0\end{array}$ & $\begin{array}{r}-7 \\
-3 \\
-4 \\
2 \\
3\end{array}$ & $\begin{array}{l}3 \\
3 \\
3 \\
4 \\
4\end{array}$ & $\begin{array}{l}86 \\
62 \\
86 \\
57 \\
83\end{array}$ & $\begin{array}{l}71 \\
70 \\
93 \\
54 \\
81\end{array}$ & $\begin{array}{r}9 \\
9 \\
9 \\
10 \\
10\end{array}$ & $\begin{array}{l}-1 \\
-6 \\
-7 \\
-2 \\
-3\end{array}$ & $\begin{array}{l}4 \\
4 \\
4 \\
4 \\
4\end{array}$ & $\begin{array}{l}72 \\
49 \\
86 \\
44 \\
74\end{array}$ & $\begin{array}{l}68 \\
54 \\
90 \\
52 \\
65\end{array}$ \\
\hline $\begin{array}{l}3 \\
4 \\
4 \\
1 \\
4\end{array}$ & $\begin{array}{r}-9 \\
1 \\
-2 \\
-3 \\
4\end{array}$ & $\begin{array}{l}3 \\
3 \\
3 \\
3 \\
3\end{array}$ & $\begin{array}{r}98 \\
125 \\
51 \\
160 \\
33\end{array}$ & $\begin{array}{r}97 \\
123 \\
43 \\
145 \\
42\end{array}$ & $\begin{array}{l}3 \\
4 \\
6\end{array}$ & $\begin{array}{l}5 \\
6 \\
0 \\
0 \\
0\end{array}$ & $\begin{array}{l}4 \\
4 \\
4 \\
4 \\
4\end{array}$ & $\begin{array}{r}158 \\
54 \\
67 \\
86 \\
42\end{array}$ & $\begin{array}{r}153 \\
64 \\
69 \\
83 \\
54\end{array}$ & $\begin{array}{l}10 \\
10\end{array}$ & $\begin{array}{l}-5 \\
-6\end{array}$ & $\begin{array}{l}4 \\
4\end{array}$ & $\begin{array}{l}73 \\
95\end{array}$ & $\begin{array}{l}71 \\
85\end{array}$ \\
\hline $\begin{array}{l}4 \\
5 \\
5 \\
5 \\
5\end{array}$ & $\begin{array}{r}9 \\
-1 \\
-2 \\
-4 \\
5\end{array}$ & $\begin{array}{l}3 \\
3 \\
3 \\
3 \\
3\end{array}$ & $\begin{array}{r}121 \\
122 \\
124 \\
91 \\
80\end{array}$ & $\begin{array}{r}108 \\
123 \\
117 \\
93 \\
77\end{array}$ & $\begin{array}{l}7 \\
9 \\
1 \\
1 \\
1\end{array}$ & $\begin{array}{l}0 \\
0 \\
1 \\
3 \\
4\end{array}$ & $\begin{array}{l}4 \\
4 \\
4 \\
4 \\
4\end{array}$ & $\begin{array}{r}83 \\
68 \\
107 \\
97 \\
90\end{array}$ & $\begin{array}{l}75 \\
69 \\
95 \\
94 \\
79\end{array}$ & & & & & \\
\hline $\begin{array}{r}-6 \\
6 \\
6 \\
6 \\
6\end{array}$ & $\begin{array}{r}2 \\
2 \\
-3 \\
-4 \\
5\end{array}$ & $\begin{array}{l}3 \\
3 \\
3 \\
3 \\
3\end{array}$ & $\begin{array}{r}57 \\
76 \\
103 \\
50 \\
64\end{array}$ & $\begin{array}{r}55 \\
81 \\
106 \\
53 \\
61\end{array}$ & $\begin{array}{r}1 \\
-1 \\
1 \\
-1 \\
2\end{array}$ & $\begin{array}{l}6 \\
7 \\
7 \\
8 \\
1\end{array}$ & $\begin{array}{l}4 \\
4 \\
4 \\
4 \\
4\end{array}$ & $\begin{array}{l}95 \\
84 \\
91 \\
80 \\
98\end{array}$ & $\begin{array}{l}85 \\
81 \\
73 \\
72 \\
97\end{array}$ & & & & & \\
\hline $\begin{array}{l}7 \\
7 \\
7 \\
7 \\
8\end{array}$ & $\begin{array}{r}1 \\
-2 \\
3 \\
-3 \\
1\end{array}$ & $\begin{array}{l}3 \\
3 \\
3 \\
3 \\
3\end{array}$ & $\begin{array}{r}83 \\
76 \\
112 \\
164 \\
130\end{array}$ & $\begin{array}{r}78 \\
71 \\
98 \\
151 \\
119\end{array}$ & $\begin{array}{l}2 \\
3 \\
3 \\
3 \\
4\end{array}$ & $\begin{array}{r}2 \\
2 \\
6 \\
-6 \\
1\end{array}$ & $\begin{array}{l}4 \\
4 \\
4 \\
4 \\
4\end{array}$ & $\begin{array}{l}59 \\
45 \\
45 \\
59 \\
99\end{array}$ & $\begin{array}{l}66 \\
53 \\
53 \\
66 \\
83\end{array}$ & & & & & \\
\hline
\end{tabular}

hedral coordination are given in table 3(A). The germanium-oxygen distances range from $1.617 \pm 0.044$ to $1.895 \pm 0.048 \AA,^{2}$ with an average value of $1.74_{0} \AA$. This agrees well with the average value of $1.74_{0} \AA$ recorded for $\mathrm{Na}_{4} \mathrm{Ge}_{9} \mathrm{O}_{20}$ (Ingri and Lundgren [5]) and with individual independent values of $1.737 \pm 0.003 \AA$ and 1.741 $\pm 0.002 \AA$ obtained by Smith and Isaacs [6] from a study of the $\alpha$-quartz form of germanium dioxide. The $\mathrm{O}-\mathrm{Ge}-\mathrm{O}$ angles vary from $94.0 \pm 3.1^{\circ}$ to $124.9 \pm 2.1^{\circ}$ with an average value of $109.3^{\circ}$.

$\mathrm{A} \mathrm{GeO}_{6}$ group is shown in figure $3,[001]$ projection. Bond distances and angles for the three independent germanium atoms in sixfold coordination are presented in table $3(\mathrm{~B})$. The mean Ge-O distance is $1.88 \AA$. This is in agreement with the value of $1.89 \AA$ given by Ondik and Smith [7] for octahedrally coordinated germanium. It is apparent that both tetrahedra and octahedra are somewhat irregular.

${ }^{2}$ Standard deviation.
Germanium-germanium distances are listed in table $3(\mathrm{C})$. The average value of $3.15_{3} \AA$ is in good agreement with the value of $3.153_{4} \AA$ obtained by Smith and Isaacs [6].

The oxygen polyhedron about the barium ion is shown in figure 4 [001] projection. Barium is coordinated to ten oxygen atoms with bond lengths ranging from $2.663 \pm 0.029 \AA$ to $3.277 \pm 0.087 \AA$. Bond distances and standard deviations are listed in table 4 . The barium ions lie in channels in the germanate network as shown in figure 1 .

\section{Relation to Benitoite}

The structural relationship between $\mathrm{BaGe}\left[\mathrm{Ge}_{3} \mathrm{O}_{9}\right]$ and $\mathrm{BaTiSi}_{3} \mathrm{O}_{9}$ proposed by Robbins and Levin [1] is essentially correct. In figure 1 , the dashed outline of the $\mathrm{BaTiSi}_{3} \mathrm{O}_{9}$ cell [3] is shown on the [001] projection of the germanate cell. Within the dashed lines, $\mathrm{Ge}(3)$ in six-fold coordination corresponds to titanium 
TABLE 3. Germanium-oxygen bond lengths and angles ${ }^{\text {a }}$

\begin{tabular}{|c|c|c|c|c|c|}
\hline \multicolumn{3}{|c|}{ Distances } & \multicolumn{3}{|c|}{ Angles } \\
\hline & $\AA$ & $\sigma, \AA$ & & & $\sigma$ \\
\hline $\mathrm{Ge}(4)-\mathrm{O}(1)$ & 1.783 & 0.039 & $\mathrm{O}(1)-\mathrm{Ge}(4)-\mathrm{O}(3)$ & $113.9^{\circ}$ & $1.8^{\circ}$ \\
\hline$O(3)$ & 1.823 & .040 & $\mathrm{O}(1)$ & 123.3 & 1.6 \\
\hline $0(6)$ & 1.617 & .044 & $\mathrm{O}(7)$ & 94.0 & 3.1 \\
\hline $\mathrm{O}(7)$ & 1.719 & .088 & $\mathrm{O}(6)$ & 96.3 & 2.1 \\
\hline & & & $O(7)$ & 105.4 & 2.6 \\
\hline & & & $\mathrm{O}(6)$ & 123.8 & 3.2 \\
\hline $\mathrm{Ge}(5)-\mathrm{O}(2)$ & 1.770 & .048 & $\mathrm{O}(2)-\mathrm{Ge}(5)-\mathrm{O}(3)$ & 97.0 & 2.0 \\
\hline $\mathrm{O}(3)$ & 1.734 & .033 & $\mathrm{O}(2) \quad \mathrm{O}(4)$ & 109.8 & 2.2 \\
\hline $\mathrm{O}(4)$ & 1.895 & .048 & $\mathrm{O}(2)$ & 124.9 & 2.1 \\
\hline $\mathrm{O}(9)$ & 1.653 & .053 & $\mathrm{O}(3)$ & 106.5 & 1.9 \\
\hline & & & $\mathrm{O}(9)$ & 109.6 & 2.6 \\
\hline & & & $\mathrm{O}(9)$ & 107.5 & 2.3 \\
\hline $\mathrm{Ge}(6)-\mathrm{O}(4)$ & 1.642 & .049 & $\mathrm{O}(4)-\mathrm{Ge}(6)-\mathrm{O}(5)$ & 108.6 & 2.8 \\
\hline $\mathrm{O}(5)$ & 1.805 & .069 & $\mathrm{O}(7)$ & 108.0 & 3.5 \\
\hline $\mathrm{O}(7)$ & 1.714 & .060 & $\mathrm{O}(8)$ & 100.0 & 2.2 \\
\hline $\mathrm{O}(8)$ & 1.720 & .042 & $\mathrm{O}(7)$ & 101.5 & 3.3 \\
\hline & & & $\mathrm{O}(8)$ & 121.9 & 2.7 \\
\hline Average & 1.740 & & $\mathrm{O}(7)$ & 116.2 & 3.1 \\
\hline & & & Average & 109.3 & \\
\hline & & & $\mathrm{Ge}(4)-\mathrm{O}(7)-\mathrm{Ge}(6)$ & 129.5 & 5.4 \\
\hline & & & $\mathrm{Ge}(4)-\mathrm{O}(3)-\mathrm{Ge}(5)$ & 122.5 & 2.3 \\
\hline & & & $\mathrm{Ge}(5)-\mathrm{O}(4)-\mathrm{Ge}(6)$ & 124.1 & 2.1 \\
\hline $\mathrm{O}(1)-\mathrm{O}(3)$ & 3.022 & .056 & & & \\
\hline$O(1)-O(6)$ & 2.993 & .059 & & & \\
\hline$O(1)-O(7)$ & 2.562 & .080 & & & \\
\hline $\mathrm{O}(3)-\mathrm{O}(6)$ & 2.566 & .047 & & & \\
\hline$O(3)-O(7)$ & 2.818 & .107 & & & \\
\hline$O(6)-O(7)$ & 2.943 & .097 & & & \\
\hline $\mathrm{O}(2)-\mathrm{O}(3)$ & 2.625 & .050 & & & \\
\hline $\mathrm{O}(2)-\mathrm{O}(4)$ & 3.000 & .071 & & & \\
\hline $\mathrm{O}(2)-\mathrm{O}(9)$ & 2.882 & .069 & & & \\
\hline $\mathrm{O}(3)-\mathrm{O}(4)$ & 2.910 & .052 & & & \\
\hline $\mathrm{O}(3)-\mathrm{O}(9)$ & 2.768 & .056 & & & \\
\hline $\mathrm{O}(4)-\mathrm{O}(9)$ & 2.865 & .068 & & & \\
\hline $\mathrm{O}(4)-\mathrm{O}(5)$ & 2.801 & .091 & & & \\
\hline $\mathrm{O}(4)-\mathrm{O}(7)$ & 2.715 & .081 & & & \\
\hline$O(4)-O(8)$ & 2.576 & .068 & & & \\
\hline $\mathrm{O}(5)-\mathrm{O}(7)$ & 2.726 & .100 & & & \\
\hline $\mathrm{O}(5)-\mathrm{O}(8)$ & 3.082 & .074 & & & \\
\hline$O(7)-O(8)$ & 2.916 & .070 & & & \\
\hline
\end{tabular}

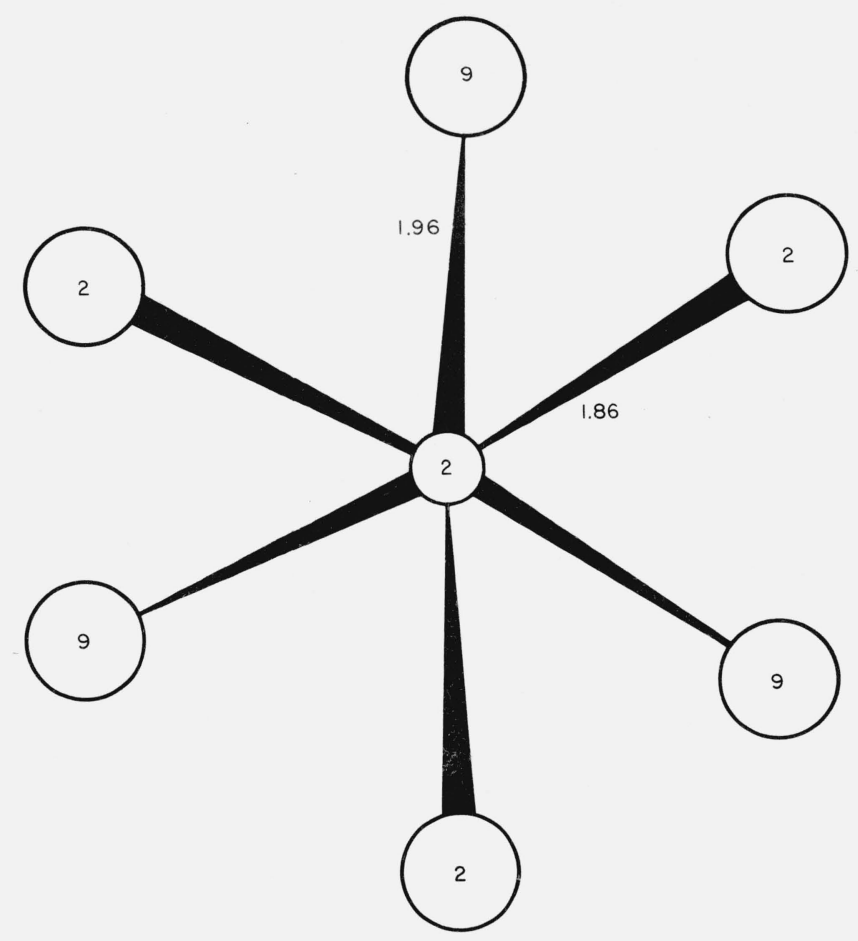

Figure 3. [001] Projection of a $\mathrm{GeO}_{6}$ group.

The numbering is consistent with that of table 1 . Bond distances are in $\AA$.

\begin{tabular}{|c|c|c|c|c|c|}
\hline \multicolumn{3}{|c|}{ Distances } & \multicolumn{3}{|c|}{ Angles } \\
\hline & $\AA$ & $\sigma, \AA$ & & & $\sigma$ \\
\hline $\mathrm{Ge}(1)-\mathrm{O}(1)$ & 1.848 & 0.036 & $\mathrm{O}(1)-\mathrm{Ge}(1)-\mathrm{O}(6)$ & $88.0^{\circ}$ & $1.5^{\circ}$ \\
\hline \multirow[t]{3}{*}{$\mathrm{O}(6)$} & 2.018 & .039 & $O(1)^{1}$ & 93.2 & 1.6 \\
\hline & & & $O(6)^{1}$ & 85.5 & 1.8 \\
\hline & & & $O(6)^{1}$ & 93.1 & 1.7 \\
\hline $\mathrm{Ge}(2)-\mathrm{O}(2)$ & 1.861 & .063 & $\mathrm{O}(2)-\mathrm{Ge}(2)-\mathrm{O}(2)^{1}$ & 94.1 & 2.5 \\
\hline \multirow[t]{3}{*}{$\mathrm{O}(9)$} & 1.957 & .051 & $\mathrm{O}(9) \quad \mathrm{O}(9)^{1}$ & 84.8 & 2.4 \\
\hline & & & $\mathrm{O}(2)^{1}$ & 94.0 & 2.0 \\
\hline & & & $\mathrm{O}(2)^{1}$ & 86.9 & 2.3 \\
\hline $\mathrm{Ge}(3)-\mathrm{O}(5)$ & 1.717 & .058 & $\mathrm{O}(5)-\mathrm{Ge}(3)-\mathrm{O}(5)^{1}$ & 91.2 & 2.8 \\
\hline \multirow[t]{2}{*}{$\mathrm{O}(8)$} & 1.858 & .056 & $\mathrm{O}(8) \quad \mathrm{O}(8)^{1}$ & 90.3 & 2.2 \\
\hline & & & $\mathrm{O}(8)^{1}$ & 89.4 & 2.3 \\
\hline Average & 1.877 & & $\mathrm{O}(5)^{1}$ & 89.4 & 2.8 \\
\hline $\mathrm{O}(1)-\mathrm{O}(6)$ & 2.688 & .059 & & & \\
\hline $\mathrm{O}(1)-\mathrm{O}(1)^{1}$ & 2.687 & .039 & & & \\
\hline $\mathrm{O}(6)-\mathrm{O}(6)^{1}$ & 2.740 & .068 & & & \\
\hline$O(1)-O(6)^{1}$ & 2.810 & .052 & & & \\
\hline $\mathrm{O}(2)-\mathrm{O}(2)^{1}$ & 2.725 & .087 & & & \\
\hline $\mathrm{O}(9)-\mathrm{O}(9)^{1}$ & 2.640 & .089 & & & \\
\hline $\mathrm{O}(2)^{1}-\mathrm{O}(9)$ & 2.793 & .080 & & & \\
\hline $\mathrm{O}(2)^{1}-\mathrm{O}(9)^{1}$ & 2.626 & .069 & & & \\
\hline $\mathrm{O}(5)-\mathrm{O}(5)^{1}$ & 2.455 & .066 & & & \\
\hline $\mathrm{O}(8)-\mathrm{O}(8)^{1}$ & 2.634 & .080 & & & \\
\hline $\mathrm{O}(8)^{1}-\mathrm{O}(5)$ & 2.516 & .084 & & & \\
\hline $\mathrm{O}(8)-\mathrm{O}(5)^{1}$ & 2.516 & .074 & & & \\
\hline
\end{tabular}

C. Distances between germanium atoms

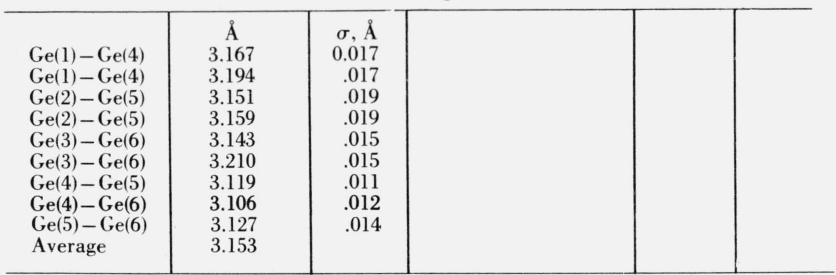

${ }^{a}$ The standard deviations are derived from the diagonal terms of the least-squares matrix.

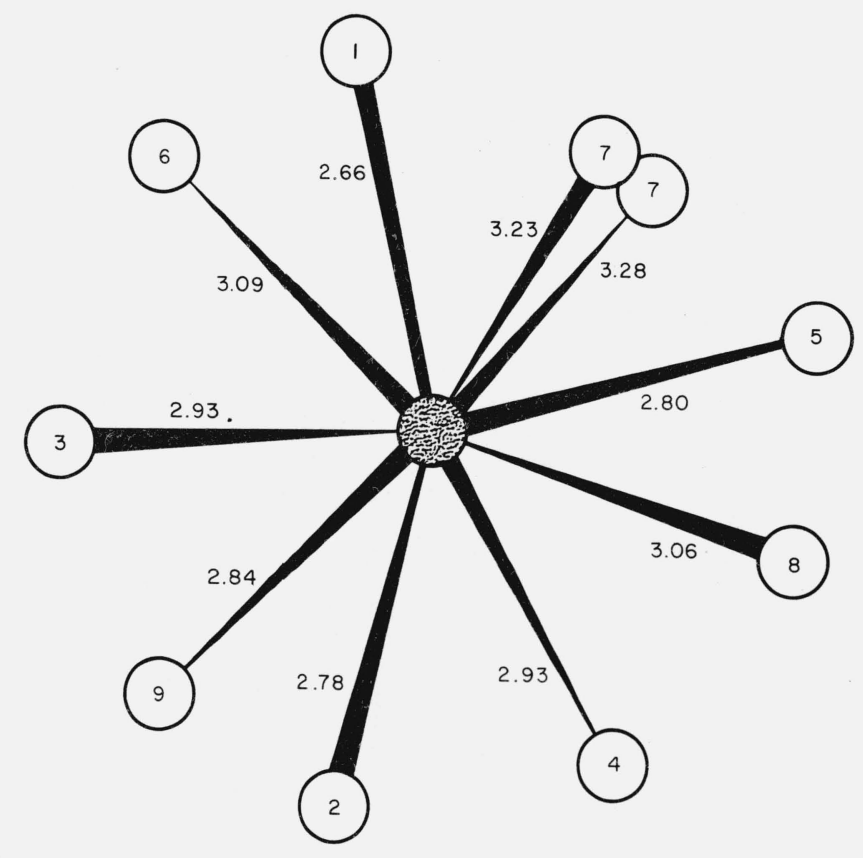

Figure 4. Nearest neighbor barium ion coordination, [001] projection.

The numbering is consistant with that of table 1 . One $O(7)$ atom is shown displaced slightly in $x$ and $y$. Bond distances are in $\AA$. 
TABLE 4. Bond distances of the barium coordination polyhedron ${ }^{\mathrm{a}}$

\begin{tabular}{r|c|c}
\hline \hline & $\AA$ & $\sigma \AA$ \\
$\mathrm{Ba}-\mathrm{O}(1)$ & 2.663 & 0.029 \\
$\mathrm{O}(6)$ & 3.088 & .035 \\
$\mathrm{O}(3)$ & 2.929 & .043 \\
$\mathrm{O}(9)$ & 2.836 & .063 \\
$\mathrm{O}(2)$ & 2.780 & .053 \\
$\mathrm{O}(4)$ & 2.927 & .032 \\
$\mathrm{O}(8)$ & 3.063 & .047 \\
$\mathrm{O}(5)$ & 2.802 & .079 \\
$\mathrm{O}(7)$ & 3.277 & .087 \\
$\mathrm{O}(7)$ & 3.227 & .087 \\
\hline \multicolumn{3}{|c}{} \\
a The standard deviations are \\
derived from the diagonal terms \\
of the least-squares matrix.
\end{tabular}

of the least-squares matrix.

in the silicate compound and the rings to $\mathrm{Si}_{3} \mathrm{O}_{9}$ groups. In the silicate cell, the origin is on a threefold axis at the center of a ring. The $\mathrm{Ba}$ ion also lies on a threefold axis. In addition, $\mathrm{BaTiSi}_{3} \mathrm{O}_{9}$ has two sheets of rings in the length of the $c$ axis $(c=9.71 \AA$, Zachariasen,

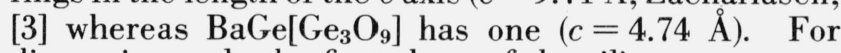
discussion, only the first sheet of the silicate structure will be considered.

With the substitution of three germanium atoms for three silicon atoms and one germanium atom for one titanium atom in the formula unit $\mathrm{BaTiSi}_{3} \mathrm{O}_{9}$ (to give $\left.\mathrm{BaGe}\left[\mathrm{Ge}_{3} \mathrm{O}_{9}\right]\right)$ the following major structural changes are noted: (1) Unlike the silicon atoms and ring oxygens of $\mathrm{Si}_{3} \mathrm{O}_{9}$, germanium atoms and ring oxygens of the $\mathrm{Ge}_{3} \mathrm{O}_{9}$ group are not coplanar and the germanium ring does not have threefold symmetry. (2) While the titanium atom lies on a threefold axis at $z=0$, three independent octahedrally coordinated germanium atoms lie on threefold axes at $z=-0.44_{0} \AA, 0.02_{9} \AA$ and $0.48_{5} \AA .^{3}$ (3) In benitoite, the barium ion is on a threefold axis surrounded by six oxygen atoms at a distance of $2.74 \AA$ and six other oxygens at a distance of $3.4 \AA$. In the germanate cell, the barium ion is linked to 10 oxygen atoms at distances ranging from $2.66 \AA$ to $3.28 \AA$, and no longer lies on a threefold axis. (4) $\mathrm{SiO}_{4}$ tetrahedra share only corners with other polyhedra in benitoite. This is true of $\mathrm{GeO}_{4}$ groups of the tetragermanate with the exception of Ge(6). The latter shares one edge with a barium polyhedron. (5) There are two sheets of rings within the $c$ dimension of benitoite whereas $\mathrm{BaGe}\left[\mathrm{Ge}_{3} \mathrm{O}_{9}\right]$ has one. Crystallographic data for the two compounds are presented in table 5.
TABLE 5. Crystal data for $\mathrm{BaGe}\left[\mathrm{Ge}_{3} \mathrm{O}_{9}\right]$ and $\mathrm{BaTiSi}_{3} \mathrm{O}_{9}$

\begin{tabular}{c|c|c|c|c|c}
\hline \hline Compound & $a, \AA$ & $c, \AA$ & $\begin{array}{c}\text { Space } \\
\text { group }\end{array}$ & Calc. density, $\mathrm{g} \mathrm{cm}^{-3}$ & $Z$ \\
\hline $\mathrm{BaGe}\left[\mathrm{Ge}_{3} \mathrm{O}_{9}\right]$ & $11.61 \pm 0.02$ & $4.74 \pm 0.01$ & $\mathrm{P} 3$ & 5.12 & 3 \\
$\mathrm{BaTiSi}_{3} \mathrm{O}_{9}{ }^{\text {a }}$ & $6.60 \pm 0.01$ & $9.71 \pm 0.01$ & $\mathrm{P} 6 \mathrm{c} 2$ & 3.73 & 2 \\
\hline
\end{tabular}

${ }^{\text {a }}$ Data from Zachariasen [3].

Computations were performed utilizing the x-ray 63 system of crystallographic programs developed at the University of Washington and the University of Maryland [8].

\section{References}

[1] C. R. Robbins and E. M. Levin, Tetragermanates of strontium, lead and barium of formula type $\mathrm{AB}_{4} \mathrm{O}_{9}$, J. Res. NBS $65 \mathrm{~A}$ (Phys. and Chem.) No. 2, 127-131 (1961).

[2] G. Eulenberger, A. Wittmann and H. Nowotny, Uber wasserfreie germanate mit zweiwertigen metallion, Monatsh 93, 123-128 (1962).

[3] W. H. Zachariasen, The crystal structure of benitoite, $\mathrm{BaTiSi}_{3} \mathrm{O}_{9}$, Z. Krist. 74, 139-146 (1930).

[4] L. H. Thomas and K. Umeda, Atomic scattering factors calculated from the TFD atomic model, J. Chem. Phys. 26, 293-303 (1957).

[5] N. Ingri and G. Lundgren, The crystal structure of $\mathrm{Na}_{4} \mathrm{Ge}_{9} \mathrm{O}_{20}$, Acta Chem. Scand. 17, 617-633 (1963).

[6] G. S. Smith and P. B. Isaacs, The crystal structure of quartzlike $\mathrm{GeO}_{2}$, Acta Cryst. 17, 842-846 (1964).

[7] H. Ondik and D. Smith, Interatomic distances in inorganic compounds, International Tables for X-ray Crystallography Vol. III, 257-274 (1962).

[8] University of Maryland, Computer Science Center, Technical Report TR-64-6 (1964). 